\title{
Article \\ Malus Antioxidant Metabolism Following Bacterial-Fungal Inoculation in Organic Farming: From Root to Fruit
}

\author{
Barbara Łata ${ }^{1, *}$, Radosław Łaźny ${ }^{1}\left(\mathbb{D}\right.$, Sebastian Przybyłko ${ }^{2}$ (D) and Dariusz Wrona ${ }^{2}$ (D) \\ 1 Laboratory of Basic Sciences in Horticulture, Institute of Horticultural Sciences, \\ Warsaw University of Life Sciences-SGGW, Nowoursynowska 159, 02-776 Warsaw, Poland; \\ radoslaw_lazny@sggw.edu.pl \\ 2 Department of Pomology and Horticulture Economics, Institute of Horticultural Sciences, \\ Warsaw University of Life Sciences-SGGW, Nowoursynowska 159, 02-776 Warsaw, Poland; \\ sebastian_przybylko@sggw.edu.pl (S.P.); dariusz_wrona@sggw.edu.pl (D.W.) \\ * Correspondence: barbara_lata@sggw.edu.pl
}

Citation: Łata, B.; Łaźny, R.;

Przybyłko, S.; Wrona, D. Malus

Antioxidant Metabolism Following

Bacterial-Fungal Inoculation in

Organic Farming: From Root to Fruit.

Appl. Sci. 2021, 11, 9466. https://

doi.org/10.3390/app11209466

Academic Editor:

Alessandra Durazzo

Received: 4 September 2021

Accepted: 9 October 2021

Published: 12 October 2021

Publisher's Note: MDPI stays neutral with regard to jurisdictional claims in published maps and institutional affiliations.

Copyright: (c) 2021 by the authors. Licensee MDPI, Basel, Switzerland. This article is an open access article distributed under the terms and conditions of the Creative Commons Attribution (CC BY) license (https:// creativecommons.org/licenses/by/ $4.0 /)$.

\begin{abstract}
This study investigated the antioxidant status of roots, leaves, and fruit upon microbial inoculation $(\mathrm{AMF}+\mathrm{PGPR}$, arbuscular mycorrhizal fungi, and plant growth-promoting rhizobacteria, respectively) of young organically farmed apple trees over two growing seasons. Three cultivars'Topaz', 'Chopin', and 'Odra'-were selected to test the relationship between inoculation and enzymatic and non-enzymatic antioxidant components. The antioxidant metabolism was highly dependent on tissue type and growing season. The greatest effect on antioxidant status following application of the inoculum was found in roots, then leaves, but it was almost negligible in fruit. Roots were influenced most by application of the inoculum in the first growing season, while leaves were influenced most in the second season. Considerable differences between the inoculated and control plants were found for root glutathione reductase (GR) and catalase (CAT) activity, as well as glutathione and ascorbate contents; root phenolics were not influenced by inoculation. In the case of leaves, effect of microbial inoculation on GR activity was revealed in the first growing season, while for global phenolics in the second season, and only the concentration of glutathione was significantly higher in the leaves of inoculated trees in both growing seasons. Leaf ascorbate content and CAT activity were not influenced by the microbial inoculation. The control and inoculated trees expressed a similar total antioxidant capacity, irrespective of the tissue type tested. Furthermore, the response of the cultivars to inoculation varied and also changed in consecutive growing seasons. Based on this study, it is likely that the effect of microbial inoculum as a tool for enhancing health-promoting properties in the fruit of perennial plants is weaker than that described for vegetables where different plant organs are edible.
\end{abstract}

Keywords: apple; arbuscular mycorrhizal fungi (AMF); plant growth-promoting rhizobacteria (PGPR) ascorbate; glutathione; glutathione reductase; phenolics

\section{Introduction}

It is widely known that there is a close relationship between a high input of fertilizers and pesticides in agro-ecosystems and numerous adverse effects, such as soil degradation, water pollution, biodiversity loss, climate change, and lastly a reduced quality of the food produced. In light of these threats, more environmentally friendly methods of soil enrichment and crop protection are being sought compared with those commonly used in agricultural practices today [1-4]. The inclusion of microorganism-based products in crop management systems due to the specific role of these rhizosphere organisms in plant performance (plant health and vigor) appears to be one option for reducing the amount of agrochemicals used [1,5-11]. Soil biota, such as plant growth-promoting rhizobacteria (PGPR) and arbuscular mycorrhizal fungi (AMF), individually and/or in co-inoculation (as a bacterial-fungal inoculum to combine the benefits of each) have been tested as a 
means of increasing the plant nutritional status [8,12-15], water status [16-18] or the host's resistance to biotic stress factors $[8,11,19]$, including soil replant disease [20]. Nitrogen fixation, phosphorus solubilization, siderophore production, a reduction in soil nutrient loss, and the effect on soil structure have been indicated to be key factors regulating nutrient uptake by plants, thus decreasing the need for chemical fertilizers $[6,11,21]$. In turn, plant nutritional status directly affects plant health and the quality of plant-derived food.

Since both the above-mentioned biotic and abiotic stresses may disturb the equilibrium between the production and scavenging of reactive oxygen species (ROS), activation of the antioxidant defense system(s) of host plants is considered to be one of the interrelationships between plants and microorganisms [17,22-24]. A plant antioxidant defense network influenced by PGPR and/or fungal inoculum can be both enzymatic and/or non-enzymatic in nature, with a direct effect on the plant chemical composition [12,25-27]. Furthermore, activation of the secondary metabolism by microorganisms may have an additional effect on the internal quality of the final products, including a greater quantity of healthpromoting phytochemicals such as flavonoids, anthocyanins, vitamins, phytoestrogens, and carotenoids $[2,7,13,28]$. The potential role of AMF in maintaining fruit quality in future climate change scenarios has also been discussed [29].

However, studies on the phytochemical content of mycorrhizal food crops still cover only a limited number of species, such as Solanum lycopersicum (tomato), Capsicum annuum (pepper), Allium cepa (onion), Cynara cardunculus (artichoke), Lactuca sativa (lettuce), Fragaria spp. (strawberry) and Zea mais (maize) [2,30]. Only a small number of cultivars have been analysed in these studies, despite cultivated species being characterized by a great number of cultivars with a high variation in internal quality traits that, in turn, are subject to the influence of several external factors [31,32]. Very little data in the literature can be found on fruit nutraceuticals due to the biofertilizer used [28] including apple [7,14], whereas the diversity and content of phytochemicals in plant products are affected by different variables [32], such as plant genotype, weather, and agronomic factors (management, soil mineral nutrients, drought, salinity, harvest season, and agrochemicals). Hence, research on microorganisms' mechanism of action and their impact on the quality of horticultural products is still deficient and at times contradictory.

Apples, the object of this study, are undisputedly a leading horticultural product worldwide, but conversion from conventional to a more sustainable or organic production currently recommended is difficult, especially due to pest and disease control [33]. Therefore, despite the notable rise in organic production in recent decades, organic farming generally still faces a number of agronomic and environmental challenges that could influence its future progress [4]. In organic farming, the use of microorganism-based products can be more effective (better root colonization by AMF/PGPR) than conventional crop management due to minimal soil disturbance and restricted use of agrochemicals [34-36].

Applications of AMF to improve growth, alleviate stress, and manage disease have been investigated in nursery apple plants, along with the beneficial effect of microorganisms on apple tree growth, nutritional status, and yield in the field [1,8,15,37-39].

However, no comprehensive study is available on the relationship between AMF and/or PGPR, and the impact of this on antioxidant status and antioxidant distribution in apple tree plant in field conditions, including organic farming. Therefore, taking different variables into account, such as apple cultivar, growing season, and tissue type, this study investigated the variability in enzymatic and non-enzymatic antioxidant components and total antioxidant capacity. It was hypothesized in particular that tissue type may be highly important after root inoculation in terms of the antioxidant metabolism and ultimately the pro-health potential of food. The other variables examined (cultivar, growing season) could also modify the effect of inoculation. Furthermore, biochemical events resulting from the interplay of bacterial-fungus inoculation and perennial plants could be of a different nature than those found in annual plants that are more frequently tested. Other benefits of this study were to test these interrelationships directly in field conditions with the full complexity of soil environment and variability in weather conditions. 


\section{Material and Methods}

\subsection{Experimental Field, Weather and Soil Conditions}

Plant material was collected from the organic experimental orchard in Warsaw at Wilanów in Central Poland $\left(52^{\circ} 9^{\prime} 36.1^{\prime \prime} \mathrm{N}+21^{\circ} 5^{\prime} 58.2^{\prime \prime} \mathrm{E}\right.$ ) in two growing seasons (2012 and 2013). The weather data were collected throughout experiment using the Vantage Pro 7 weather station (Davis, CA, USA) and are presented in Figure 1. Average temperatures in the particular months of both growing seasons were similar, however the sum of precipitation in 2013 was considerably higher than in 2012, although there was no precipitation in July 2013. The plants of three cultivars recommended for organic farms- 'Topaz', 'Chopin', and 'Odra' - were planted in 2011 on M.9 rootstock on a silty-loam alluvial soil within a split-block experimental design with four replications (three trees per replication). The plants were grown in rows spaced $3.5 \times 2 \mathrm{~m}$ apart, and trained on a spindle bush training system. The control (N-INO, non-inoculated trees) and inoculated trees (INO) with AMF (mycorrhizal arbuscular fungi) + PGPR (plant growth-promoting rhizobacteria) were considered. Commercially available microbial inoculum Micosat F (CCS Aosta, Quart, Italy) at a total concentration of $106 \mathrm{CFU} \mathrm{g}^{-1}$, containing ground and shredded roots of host plants with spores and mycelium of AMF Glomus mosseae GP11, G. viscosum GC41, G. intraradices GB67, and PGPR Bacillus subtilis BA41 and Streptomyces spp. SB19 formulated as a powder, were applied to $30 \mathrm{~cm}$-deep soil pits during tree planting at $10 \mathrm{~g}$ tree $\mathrm{e}^{-1}$ dose. Then in each year of the experiment, a $2 \mathrm{~g} \mathrm{~m}^{-2}$ dose of the inoculum was applied next to the tree three times per season at three-week intervals, with the first dose applied at the beginning of May. Agrotechnical treatments were maintained in accordance with the recommendations for organic farming. More details on the experimental design can be found in the paper recently published by Przybyłko et al. [15].

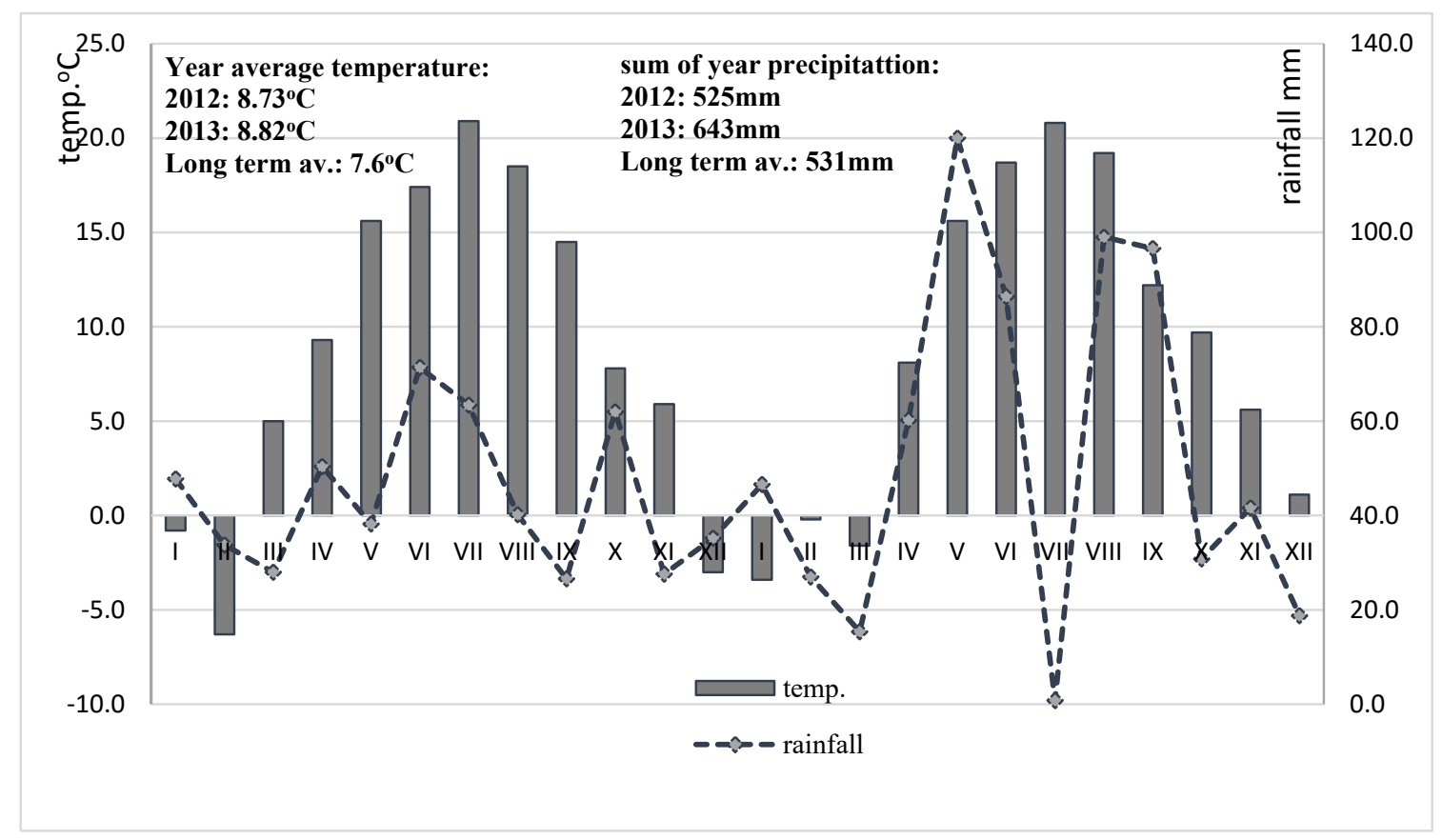

Figure 1. Data on mean monthly temperatures and rainfall at the experimental site in the 2012 and 2013 growing periods. Long term averages (av.) were calculated for the period 1982-2012.

\subsection{Root, Leaf and Fruit Sample Collection and Analysis}

Root samples were collected each year at the end of June from a depth of up to $30 \mathrm{~cm}$ using a field spade, and then were transferred to the laboratory and cleaned under tap water. The parameters of mycorrhizal frequency (the share of root fragments in which mycorrhizal structures have formed), relative mycorrhizal intensity (the share of the colonized root fragment area in which mycorrhizal structures have developed), and 
the absolute intensity of mycorrhiza (the share of the whole root fragment area in which mycorrhizal structures have developed) were evaluated. These results have recently been published by Przybyłko et al. [15]. In summary, in both growing seasons inoculated apple tree roots were characterized by a significantly higher value of all parameters regarding mycorrhizal frequency, without large differences between cultivars [15]. Some of the collected roots were intended for antioxidant analysis. Leaf samples for antioxidant analysis were collected at the same time as the roots. Each sample consisted of 10-15 similarly sized leaves located at the same height of the canopy, gathered from shoots on both sides of every plant, and had an appearance typical for the particular treatment.

Apples were harvested in both years during the first ten days of October. Based on a previous comprehensive study [40] that reveals apple peel to be a good marker of health values of the whole apple fruit (apple antioxidants are mainly located in the peel, as well as there being a high correlation coefficient between apple peel and whole fruit antioxidant concentrations), this part of the fruit was collected and tested, six apples per replication were peeled and immediately put into liquid nitrogen.

Analyzed samples of different tissue type were prepared in four replications, each of them consisting of material taken from three trees from each block.

The collected plant samples were frozen in liquid nitrogen and transferred to a deep freeze at $-80^{\circ} \mathrm{C}$. Root, leaf, and fruit tissue were ground in mortars (approximately 20-25 g in each replicate and tissue type) with liquid nitrogen to fine powder before analysis and the tissue powder was used for antioxidant determination.

\subsection{Antioxidant Measurements}

Activity of glutathione reductase (GR) and catalase (CAT). The root, leaf, and fruit tissue powder was weighed $(200 \mathrm{mg}$ ) and suspended in $100 \mathrm{mM}$ of potassium phosphate buffer (5 mL, pH 7.8) containing Triton X-100 (0.5\%), insoluble polyvinylpolypyrrolidone (PVPP), and ascorbate $(5 \mathrm{mM})$. The mixture was centrifuged at $48,000 \times g$ for $20 \mathrm{~min}$ at $4{ }^{\circ} \mathrm{C}$ (refrigerated centrifuge 3-30KS, Sigma, Osteorode, Germany). Enzyme activity (GR: EC 1.6.4.2, CAT: EC 1.11.1.6) was measured spectrophotometrically (HITACHI UV-Vis spectrophotometer, model U2900, Kyoto, Japan) by monitoring the decrease in absorbance at $240 \mathrm{~nm}$ and $340 \mathrm{~nm}$ for CAT and GR, respectively [31].

Enzyme activity was expressed in nanokatals per gram fresh weight (nkat g ${ }^{-1} \mathrm{FW}$ ).

Thiol compounds and ascorbate. Powdered plant tissue was weighed and homogenized in $0.1 \mathrm{M}$ of $\mathrm{HCl}$ containing PVPP (refrigerated centrifuge, Micro 22R, Hettich, Tuttlingen, Germany). The content of glutathione and L-cysteine compounds was determined using high-performance liquid chromatography (HPLC) with a Waters 474 Scanning Fluorescence Detector (Waters Co., Milford, MA, USA; System Breeze with a binary solvent delivery system (1525), a degasser, an autosampler with thermostat (M717 PLUS) and the thermostat (Peltier) for the column)), using a Luna C18 $100 \AA$ column $(150 \times 4.6 \mathrm{~mm}, 5 \mu \mathrm{m}$, Phenomenex, Torrance, CA, USA) for separation [41]. The total glutathione (tGSH: GSH + GSSG, reduced and oxidized forms respectively) and L-cysteine contents were measured in the same supernatant after reduction with dithiothreitol (DTT) and derivatization with monobromobimane. Separation was carried out by applying a solution of $10 \%$ methanol (solvent $\mathrm{A}, \mathrm{pH} 4.3$ ) and $90 \%$ methanol containing $0.25 \%(v / v)$ glacial acetic acid (solvent $\mathrm{B}$, $\mathrm{pH}$ 3.9). The flow rate was $1 \mathrm{~mL} \mathrm{~min}^{-1}$.

The total concentration of ascorbate (L-AA + DHAA; L-ascorbic and dehydroascorbic acids, respectively) was measured after complete reduction of DHAA with DTT [41]. Separation was carried out using an Atlantis ${ }^{\mathrm{TM}} \mathrm{dC}_{18}$ column $(250 \mathrm{~mm} \times 4.6 \mathrm{~mm}, 5 \mu \mathrm{m}$, Waters Co., Milford, MA, USA) coupled with a UV-VIS detector (Waters 2487) at $268 \mathrm{~nm}$ under isocratic conditions. The mobile phase contained $10 \%$ methanol and $2 \% \mathrm{NH}_{4} \mathrm{H}_{2} \mathrm{PO}_{4}$ with a $\mathrm{pH}$ of 2.8 . The flow rate was $1 \mathrm{~mL} \mathrm{~min}^{-1}$.

The concentrations of the above compounds were calculated using standard calibration curves. 
Total phenolics content (TPC). TPC was evaluated using the Folin-Ciocalteu method [42] with a two-step extraction with methanol in an ultrasonic bath [43]. The TPC was expressed in gallic acid equivalents (GAE).

Trolox equivalent antioxidant capacity (TEAC). Three different tests were applied to measure TEAC: FRAP, ABTS, and DPPH assays. These methods have previously been described [41]. The results were calculated using a standard calibration curves and are expressed as mmol TE kg-1 FW (TE-Trolox equivalents).

Reagents. GSH, L-AA, DTT, TE, and gallic acid were from Sigma (St. Louis, MO, USA), L-Cysteine was purchased from Fluca (Buchs, Switzerland). Monobromobimane (thiolyte) was from Calbiochem Co. All other chemicals were of analytical or HPLC gradepurity.

\subsection{Statistical Analysis and Presentation of Data}

The data obtained were elaborated using multifactor analysis of variance (ANOVA) within the Statistica 13 software package (StatSoft, Cracow, Poland). Due to distinct weather conditions in each season, each year's results were elaborated separately. The significance of differences between the means was evaluated using Tukey's test at the 5\% probability level.

Since inoculation did not affect total antioxidant capacity, detailed data concerning TEAC based on the ABTS, DPPH, and FRAP tests are presented as supplementary information in Table S1 (see Supplementary Materials).

\section{Results}

\subsection{Statistical Analysis}

Statistical analysis revealed the greater impact of inoculation on root antioxidant properties compared to leaves and fruits (Table 1). In both growing seasons the effect of inoculation has been statistically proved on the content of root ascorbate, glutathione, and GR activity at a very high probability level $(\alpha=0.001)$. The interaction between main factors (cultivar $\mathrm{x}$ inoculation) depended on the year as well as component tested. Based on F value the interaction was, in general, weaker compared to the effect of main factors. Influence of inoculation on leaf antioxidant properties was proved in case of glutathione, GR and CAT activity in 2012, while in 2013 for L-cysteine, glutathione, and phenolics (Table 1). Except for leaf GR activity, in the second growing season interaction between cultivar and applied bacterial-fungal inoculum occurred at a high probability level $(\alpha=0.01)$ for the most component tested. In the case of fruit it can be concluded that there was no impact of inoculation on the antioxidant tested (an exception was CAT activity in 2012). The cultivar effect on antioxidant properties was very variable depending on the year, tissue type as well as component tested. Based on F value and probability level (Table 1) cultivar effect on the root antioxidant properties was comparable in both growing seasons. In case of leaves, cultivar effect was more pronounced in the second growing season. Cultivar effect on fruit non-enzymatic antioxidants (ascorbate, glutathione, phenolics) in both growing seasons at high and very high probability level was revealed. In turn, cultivar effect on fruit antioxidative enzyme activity ranged from non-significant in the first growing season to a significant at a very high probability level in the subsequent one. 
Table 1. Summary of analysis of variance (ANOVA) for the components tested. Values of $\mathrm{F}$ for particular sources of variation and their significance depending on the growing season, tissue type, and component tested.

\begin{tabular}{|c|c|c|c|c|c|c|c|c|c|c|c|c|}
\hline \multirow{5}{*}{ Component } & \multicolumn{12}{|c|}{ Growing Season } \\
\hline & \multicolumn{6}{|c|}{2012} & \multicolumn{6}{|c|}{2013} \\
\hline & \multicolumn{12}{|c|}{ Source of Variation } \\
\hline & \multirow{2}{*}{\multicolumn{2}{|c|}{ Cultivar (A) }} & \multirow{2}{*}{\multicolumn{2}{|c|}{ Inoculation (B) }} & \multirow{2}{*}{\multicolumn{2}{|c|}{$\begin{array}{c}\text { Interaction: } \\
\mathrm{AB}\end{array}$}} & \multirow{2}{*}{\multicolumn{2}{|c|}{ Cultivar (A) }} & \multirow{2}{*}{\multicolumn{2}{|c|}{ Inoculation (B) }} & \multicolumn{2}{|c|}{ Interaction: } \\
\hline & & & & & & & & & & & \multicolumn{2}{|c|}{$A B$} \\
\hline df & \multicolumn{2}{|c|}{2} & \multicolumn{2}{|c|}{1} & \multicolumn{2}{|c|}{2} & \multicolumn{2}{|c|}{2} & \multicolumn{2}{|c|}{1} & \multicolumn{2}{|c|}{2} \\
\hline \multicolumn{13}{|c|}{ ROOT } \\
\hline $\mathrm{L}-\mathrm{AA}+\mathrm{DHAA}$ & 30.2 & $* * *$ & 15.8 & $* * *$ & 7.62 & $* *$ & 30.2 & $* * *$ & 74.6 & $* * *$ & 8.47 & ** \\
\hline L-Cys & 9.49 & $* *$ & 25.5 & $* * *$ & 3.65 & $*$ & 6.96 & $* *$ & 2.00 & ns & 0.99 & ns \\
\hline GSH+GSSG & 1.05 & ns & 25.2 & $* * *$ & 4.60 & $*$ & 6.82 & $* *$ & 19.7 & $* * *$ & 8.10 & ** \\
\hline $\mathrm{TPC}$ & 1.17 & ns & 0.02 & ns & 0.95 & ns & 0.94 & ns & 0.06 & ns & 0.30 & ns \\
\hline GR & 618 & $* * *$ & 87.6 & $* * *$ & 9.49 & $* *$ & 9.03 & $* *$ & 43.7 & $* * *$ & 4.14 & * \\
\hline CAT & 13.7 & $* * *$ & 19.2 & $* * *$ & 0.70 & ns & 14.3 & $* * *$ & 5.97 & $*$ & 6.21 & $* *$ \\
\hline \multicolumn{13}{|c|}{ LEAF } \\
\hline $\mathrm{L}-\mathrm{AA}+\mathrm{DHAA}$ & 0.58 & ns & 1.67 & ns & 4.88 & * & 11.8 & $* * *$ & 0.34 & $\mathrm{~ns}$ & 5.16 & * \\
\hline L-Cys & 6.35 & $* *$ & 0.68 & ns & 0.24 & ns & 26.5 & $* * *$ & 29.3 & $* * *$ & 8.27 & ** \\
\hline $\mathrm{GSH}+\mathrm{GSSG}$ & 1.94 & ns & 6.25 & * & 0.23 & ns & 28.1 & $* * *$ & 24.3 & $* * *$ & 6.81 & ** \\
\hline TPC & 1.47 & ns & 0.57 & ns & 0.39 & ns & 8.82 & $* *$ & 6.46 & * & 7.16 & ** \\
\hline GR & 5.41 & $*$ & 11.3 & $* *$ & 0.29 & ns & 32.4 & $* * *$ & 1.99 & $\mathrm{~ns}$ & 2.01 & ns \\
\hline CAT & 4.47 & $*$ & 7.02 & * & 1.53 & ns & 11.9 & $* * *$ & 3.05 & ns & 7.37 & $* *$ \\
\hline \multicolumn{13}{|c|}{ FRUIT } \\
\hline $\mathrm{L}-\mathrm{AA}+\mathrm{DHAA}$ & 10.5 & $* * *$ & 0.07 & ns & 6.79 & $* *$ & 19.9 & $* * *$ & 1.68 & ns & 0.99 & ns \\
\hline L-Cys & 26.7 & $* * *$ & 2.55 & ns & 0.45 & ns & 0.89 & ns & 0.00 & ns & 1.79 & ns \\
\hline GSH+GSSG & 23.9 & $* * *$ & 2.85 & ns & 0.55 & ns & 7.89 & $* *$ & 0.00 & $\mathrm{~ns}$ & 0.33 & ns \\
\hline $\mathrm{TPC}$ & 8.61 & $* *$ & 0.55 & ns & 2.48 & ns & 10.6 & $* * *$ & 3.25 & ns & 4.35 & * \\
\hline GR & 2.34 & ns & 0.09 & ns & 1.95 & ns & 38.4 & $* * *$ & 0.00 & ns & 0.18 & ns \\
\hline CAT & 25.7 & $* * *$ & 6.92 & ** & 1.58 & ns & 1.36 & ns & 0.14 & $\mathrm{~ns}$ & 0.14 & $\mathrm{~ns}$ \\
\hline
\end{tabular}

${ }^{* * *}$ significant at $\alpha=0.001 ;{ }^{* *}$ significant at $\alpha=0.01 ;{ }^{*}$ significant at $\alpha=0.05 ;$ ns-non-significant; L-AA+DHAA: total ascorbate content (the sum of the reduced (L-AA) and oxidized (DHAA) forms; L-Cys: L-cysteine; GSH+GSSG: total glutathione content the sum of the reduced (GSH) and oxidized (GSSG) forms; TPC: total phenolics; GR: glutathione reductase; CAT: catalase.

\subsection{Antioxidant Enzyme Activity upon Bacterial-Fungal Inoculation: From Root to Fruit}

Root. Statistical analysis revealed a significant influence of apple tree inoculation on root GR and CAT activities, irrespective of the growing season (Tables 1 and 2). A clearer, unequivocal response in the case of GR activity was found. Inoculated plants exhibited approximately twice the GR activity, based on mean values, in both tested years compared with the control plants (Table 2). Furthermore, the inoculation effect (differences between inoculated plants and control plants expressed in percentage) on GR activity depended greatly on the cultivar. The increase in GR activity ranged from 57\% ('Chopin') to $141 \%$ ('Topaz') and from 62\% ('Odra') to 163\% ('Chopin') in 2012 and 2013, respectively. The inoculation effect in the case of the 'Chopin' cultivar increased between 2012 and 2013, while it decreased for the 'Topaz' and 'Odra' cultivars. CAT activity in the roots of INO plants was significantly higher in 2012, ranging from $11 \%$ ('Odra') to 22\% ('Topaz'). Although on average in the following year there was an increase in CAT activity in the roots of control plants (non-inoculated), the inoculation effect differed depending on the cultivar. Root CAT activity of treated 'Topaz' was higher than the control trees, whereas in the case of 'Odra', there was a significant reduction in CAT activity in roots of inoculated plants. These differences were weak in 'Chopin' however. 
Table 2. Root, leaf, and fruit antioxidant enzyme activity: year, cultivar, and bacterial-fungal inoculation effects.

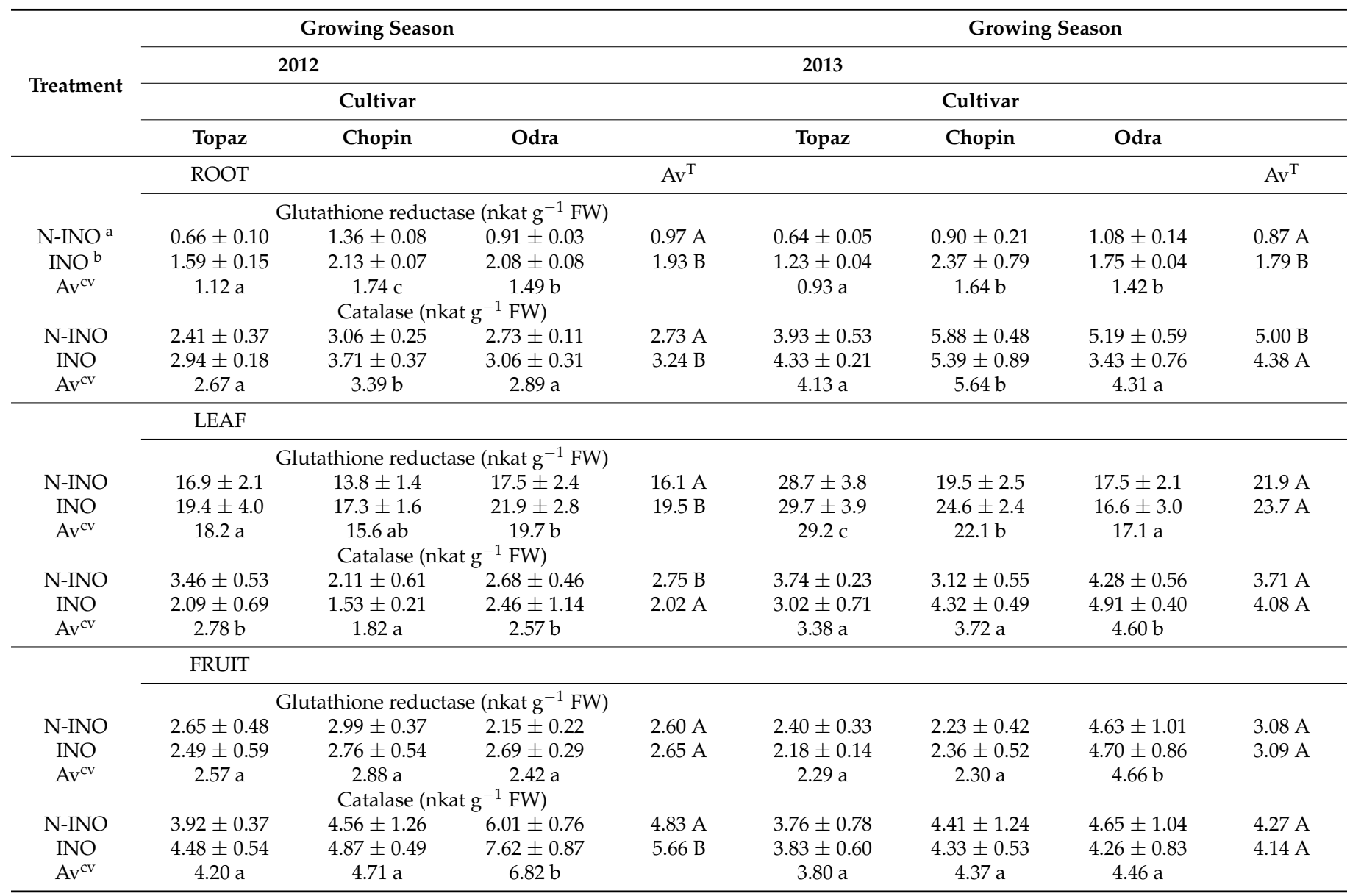

${ }^{a}$ non-inoculated plants; ${ }^{\mathrm{b}}$ inoculated plants; mean separation for treatment (N-INO vs. INO plants) and cultivar by Tukey test ( $\left.p<0.05\right)$; means followed by the same letter in column ( $A v^{\mathrm{T}}$ —average for treatment) and in line (Av ${ }^{\mathrm{cv}}$-average for cultivar) do not differ significantly, each year's results were elaborated separately; presented values are the means of four replicates, \pm SD; FW—fresh weight.

Leaves. A significant influence of inoculation on GR activity was identified in 2012, with leaf GR activity increasing in treated plants from 15\% ('Topaz') to 25\% ('Chopin', 'Odra') compared with the leaves of the control trees (Table 2). In the following year, this effect was maintained in the case of the 'Chopin' cultivar only. Other cultivars expressed a similar level of leaf GR activity irrespective of the treatments. In contrast to GR, the CAT activity in the leaves of inoculated plants decreased significantly in 2012 (Table 2). All the examined cultivars expressed a similar behavior, with the largest drop exhibited by 'Topaz' and the smallest by 'Odra'. In turn, in 2013, an increase in CAT activity in the leaves of treated apple trees was revealed in the 'Chopin' and 'Odra' cultivars, while the leaves of the 'Topaz' control plants, similarly to 2012, were characterized by a greater activity of this enzyme.

Fruit. Glutathione reductase activity in the apple peel of non-inoculated and inoculated plants was similar in both growing seasons (Table 2). 'Odra' expressed a $25 \%$ greater peel GR activity in treated plants, but only in 2012. Compared with the control plants, CAT activity in the apple peel of inoculated plants was significantly higher in 2012 (17\% more on average). However, the colonization effect on CAT activity depended greatly on the cultivar. The increase in CAT activity of INO plants ranged from $7 \%$ ('Chopin') to $27 \%$ ('Odra'). This effect was not visible in 2013, where the apple peel of the control plants and treated plants exhibited nearly the same CAT activity. 


\subsection{Non-Enzymatic Antioxidants upon Bacterial-Fungal Inoculation: From Root to Fruit}

Root. Except for global phenolic content, an inoculation effect was revealed for nonenzymatic antioxidants, and the influence due to the inoculum applied was more distinct in the first of the growing seasons examined (Tables 1 and 3). In 2012, the roots of inoculated plants had a significantly higher content of total ascorbate and glutathione, as well as of L-cysteine, the precursor of glutathione. In 2013, only the concentration of glutathione was significantly higher in INO plants.

In general, all the cultivars expressed a fairly similar pattern of antioxidant changes under the influence of the inoculum applied, but the size of the inoculation effect differed greatly. As can be seen (Table 3), in 2012 the greatest differences between the control and inoculated plants were in 'Topaz' (36\%), 'Odra' $(150 \%)$, and 'Chopin' $(278 \%)$, while the smallest differences for 'Odra' ( $7 \%)$, 'Chopin' (15\%), and 'Topaz' (33\%) related to root total ascorbate, L-cysteine, and total glutathione concentrations, respectively (Table 3). The effect of inoculation, although much weaker for L-cysteine, was still present in the case of glutathione in the consecutive growing season. In contrast to 2012, the ascorbate content in 2013 decreased significantly in the roots of inoculated plant trees. Consequently, irrespective of the growing season, the applied inoculum did not influence the concentration of root phenolic compounds.

Table 3. Root, leaf, and fruit non-enzymatic antioxidant content: year, cultivar, and bacterial-fungal inoculation effects.

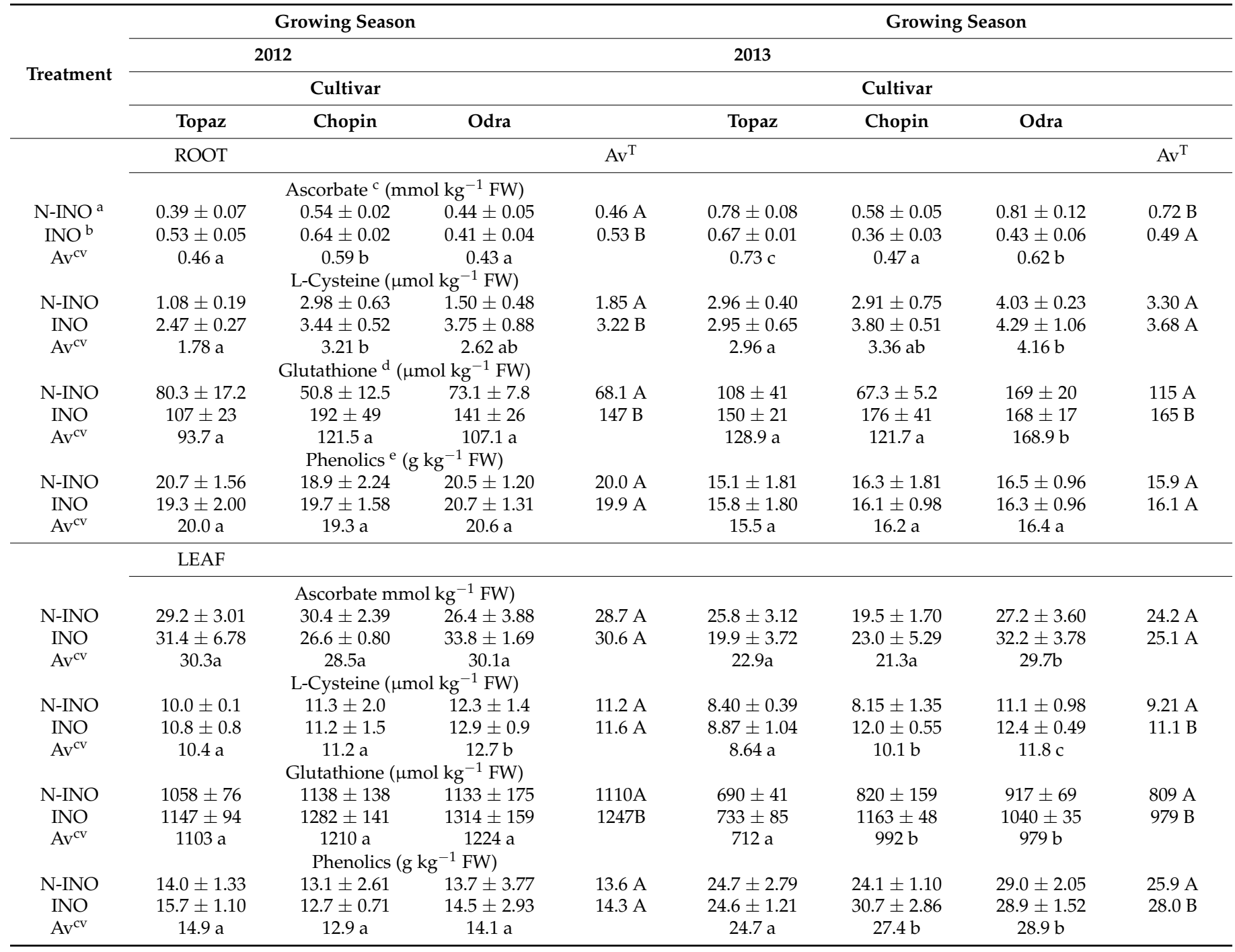


Table 3. Cont.

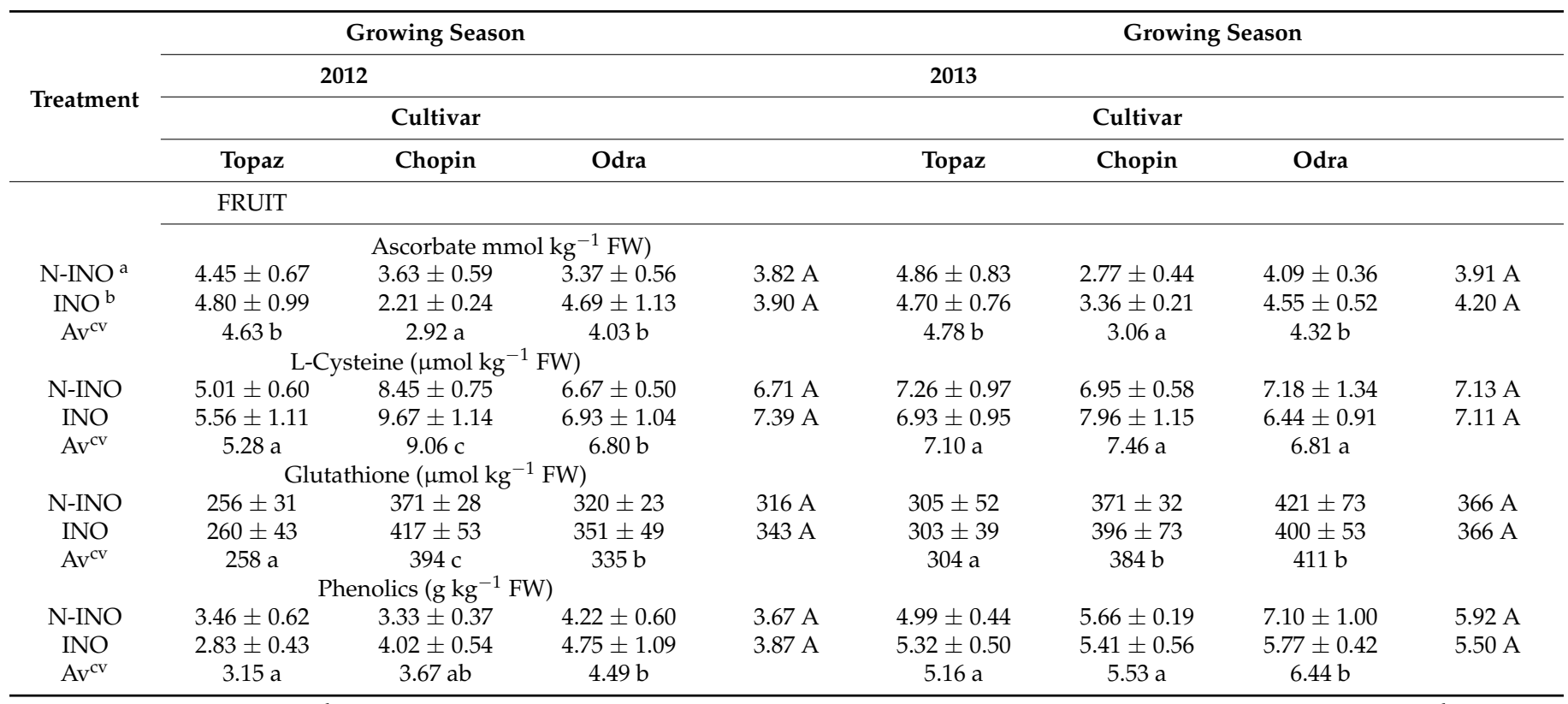

${ }^{\mathrm{a}}$ non-inoculated plants; ${ }^{\mathrm{b}}$ inoculated plants; ${ }^{\mathrm{c}}$ total ascorbate content $=$ the sum of the reduced (L-AA) and oxidized forms (DHAA); ${ }^{\mathrm{d}}$ total glutathione content $=$ the sum of the reduced (GSH) and oxidized forms (GSSG); ${ }^{\mathrm{e}}$ global phenolics expressed in gallic acid equivalent; mean separation for treatment (N-INO vs. INO plants) and cultivar by Tukey test $(p<0.05)$; means followed by the same letter in column $\left(\mathrm{Av}^{\mathrm{T}}\right.$-average for treatment) and in line ( $\mathrm{Av}^{\mathrm{cv}}$-average for cultivar) do not differ significantly, each year's results were elaborated separately; presented values are the means of four replicates, \pm SD; FW-fresh weight.

Leaves. In the first growing season (2012), only the glutathione concentration was significantly higher in the leaves of treated plants (Table 3). A stronger AMF/PGPR effect occurred in 2013, where on average there was a significant increase both in thiol and phenolic compounds in the leaves of inoculated plants. However, looking at individual cultivars, the inoculation effect was very variable both regarding the analyzed compounds and growing season. The increase in glutathione concentration in the leaves of the treated plants ranged from $8 \%$ ('Topaz') to $16 \%$ ('Odra') in 2012 and from $6 \%$ ('Topaz') to $42 \%$ ('Chopin') in 2013. In the case of phenolic compounds, it can be concluded that the inoculation effect in 2012 was revealed in 'Topaz' (concentration increased by $12 \%$ ) and in 2013 in the 'Chopin' cultivar (concentration increased by 27\%). Although statistical analysis did not reveal the effect of bacterial-fungal inoculation on the concentration of ascorbate in leaves, in the case of 'Odra' there was a meaningful increase in the content of this compound of $28 \%$ and $18 \%$ in 2012 and 2013 , respectively.

Fruit. The influence of applied inoculum on apple peel non-enzymatic antioxidant content was not proven statistically (Tables 1 and 3). However, some increases in the most tested antioxidants in the apple peel of inoculated plants of the 'Chopin' cultivar were observed. In 2012, L-cysteine, glutathione and global phenolics were 14, 12 and 21\% higher respectively, while in 2013 there was also a large increase in ascorbate concentration (21\%). A great increase in the ascorbate content of the apple peel of inoculated 'Odra' plants was also noted, especially in the first growing season (39\%).

The total antioxidant capacity (TAC) using ABTS, DPPH, and FRAP tests was evaluated in different tissue type and growing seasons. The TAC determined in root tissue did not differ between inoculated and non-inoculated plants (Table S1). Some differences between growing seasons were noted for ABTS and DPPH, whose values increased considerably in 2013 compared with 2012. The TAC of leaf tissue did not differ between inoculated and non-inoculated plants. As in the case of root tissue, a difference between growing seasons was noted. Compared with 2012, TEAC values increased in 2013 by 27, 32, and $40 \%$ for FRAP, ABTS, and DPPH, respectively. There were no differences between inoculated and non-inoculated apple trees in the TAC values of apple peel. Therefore, detailed data 
on TEAC based on the ABTS, DPPH, and FRAP tests are presented as supplementary information in Table S1 (see Supplementary Materials).

\section{Discussion}

One challenge facing contemporary horticulture is to ensure a sufficient amount of high-quality food for the world's growing population without aggravating existing environmental threats [44]. Health-promoting and protecting attributes of phytochemicals in edible horticultural crops have also led to an intensive search for different ways of increasing their contents. One promising approach in sustainable intensification seems to be the use of microorganism-based products called biofertilizers/biostimulants $[7,10,13,28]$.

AMF or the co-inoculation of PGPR and AMF of plant roots intensifies the oxidant scavenging system in host plants, and thus minimizes damage at a cellular level in stressful conditions [45]. Thus, inoculated plants exposed to biotic or abiotic stresses display lower hydrogen peroxide $\left(\mathrm{H}_{2} \mathrm{O}_{2}\right)$ and superoxide $\left(\mathrm{O}_{2}\right)$ contents and lower lipid peroxidation with a simultaneous rise in enzymatic and/or non-enzymatic antioxidants compared with control plants $[16,19,22,24,46]$. An increase in the content of antioxidants in inoculated plants is also reported as a possible way to enhance the biological value of crop plants. Papers summarizing research on the role of AMF and PGPR in shaping health-promoting properties of plant products have recently been published [2,30]. Investigations on phytochemical contents in food crops as affected by bacterial/fungal symbiosis have been limited to a few plant species and cultivars. These were mainly pot experiments in specific production systems, such as greenhouse conditions, and with vegetables as the test plants [2,44]. Due to differences in the experimental designs, including differences in the microorganisms used, different effects have been observed. Therefore, understanding of the mechanism of action of these microorganisms and their impact on the quality of horticulture products is still lacking and contradictory. Field research containing all the complexity of the soil environment as well as variable weather conditions is even more of a challenge. In addition to the above-mentioned factors, another difficulty when testing perennial plants, especially trees, is plant age, which influences tree physiology.

The present study attempted to evaluate selected elements of the antioxidant machinery following application of a bacterial-fungal inoculum for organically grown Malus domestica in field conditions. Roots, leaves, and fruits were evaluated to establish a possible site effect due to the introduction of inoculum to the rhizosphere. Different cultivars and growing seasons were considered as coexisting factors.

In this study, GR and CAT were selected as enzymatic markers of the possible interaction between apple trees and applied inoculum, and of a possible occurrence of oxidative stress. Among other things, these enzymes are involved in the removal of $\mathrm{H}_{2} \mathrm{O}_{2}$, one of the first metabolites in gradual oxygen reduction [24]. In the case of GR, removal takes place via the glutathione-ascorbate cycle (also examined in this study), while CAT is directly involved in $\mathrm{H}_{2} \mathrm{O}_{2}$ removal. Previous studies on a very large group of apple cultivars reveal GR to be an enzyme that is highly sensitive to environmental conditions [31]. The activity of GR in inoculated trees was significantly greater in both growing seasons in root tissues, and in leaves in the first growing season, while GR was not affected in apple peel. On average, the roots of inoculated trees exhibited double the GR activity. However, the level of increase in GR activity in tested cultivars was strictly related to the growing season, with large differences between the control and treated plants in GR activity noticed in the first year of the study, which lessened in the subsequent season. Similarly, the poor GR response to the inoculum in the first year was stronger in the subsequent growing season. Hence, the year effect in perennial plants may be of greater importance and increase the difficulty of interpreting the results than is the case in annual plants. With reference to the above, the 'Topaz' and 'Odra' cultivars displayed the greatest increase in root GR activity in the first of the seasons tested, while this was the true of 'Chopin' in the second season. The same regularity was recorded in the case of leaf GR activity in 'Topaz' and 'Odra', while 'Chopin' in both the years studied expressed the same differences in leaf 
GR between inoculated and non-inoculated plants. Cultivar differences in mycorrhizal frequency, absolute mycorrhizal intensity, and relative mycorrhizal intensity were more noticeable in 2012 than in 2013 [15]. In fact, in 2013 there were no cultivar differences in mycorrhizal parameters [15]. Thus, in addition to the inoculation effect, the age of the plants and year effect could be involved in tree antioxidant expression or could be related to differences in the endogenous antioxidant levels of cultivars. The large number of apple cultivars available on the market also means a broad range of variability in quality traits. Lastly, a substantial variation can be expected due to agrotechnical approaches, including the effects of biofertilizers [31,32].

The picture of changes in CAT activity was more inconsistent than those in GR activity. The roots and apple peel of inoculated apple trees expressed significantly higher CAT activity in the first growing season only, irrespective of the cultivar examined. This effect was not found in 2013. Leaf CAT activity was considerably higher in control plants in 2012, while in 2013 the increase in CAT activity due to inoculation was exhibited in 'Chopin' and 'Odra' leaves.

Several studies describe the relationship between enzymatic antioxidants such as GR, CAT, SOD, APX, POD and plant AMF/PGPR symbioses [12,16,17,22,23,26]. It should be noted that in these studies the microbial inoculation effect on antioxidative enzyme activity was investigated in relation to various abiotic stresses, such as drought [22], salinity [12,26], water stress [16,17], and herbicide [23].

Porcel et al. [22] emphasize that of several enzymes tested (APX, SOD, CAT, GR), only GR activity was higher in roots and the nodule of mycorrhizal soybean plants than in non-mycorrhizal plants. Mycorrhizal citrus roots in a pot culture demonstrated an increase in GR activity [16]. In turn, AMF colonization was accompanied by an enhancement of activity CAT in the leaves of Lycopersicon esculentum L. [12], Elaeagnus angustifolia L. [26], and Olea europaea [17]. Inconsistent results regarding leaf CAT activity as an effect of AMF in soybean plants and oxidative stress responses generated by paraquat have been reported by Bressano et al. [23].

As the increase in enzyme activity in inoculated plants is frequently accompanied by lower levels of malondialdehyde (MDA), $\mathrm{H}_{2} \mathrm{O}_{2}$, and superoxide $\left(\mathrm{O}_{2}.\right)$, an assumption was made that AMF mitigates oxidative stress due to the activation of several enzymatic antioxidants in an adverse environment [16,17]. Assuming that organic production generates several stresses since this system excludes agrochemical applications [36], the presented results also seemed to confirm the above statement. No research on apple trees could be found that was more directly comparable with the results presented in this study.

Higher activity of antioxidant enzymes is frequently accompanied by enhanced synthesis of non-enzymatic antioxidants that are important for both plant and human health due to their involvement in oxidative stress regulation and many other regulatory functions. The beneficial effects of mycorrhizal symbiosis on the yield and fruit quality of crops, however, can vary depending on internal (plant genotype) and external factors (overall experiment conditions) as well as fungal strains $[2,29,30]$.

The ascorbate-glutathione (AsA-GSH) pathway comprises these two antioxidants and four enzymes-APX, MDHR, DHAR, and GR - that play a key role in ROS detoxification and interact with other plant defense systems to protect plants from oxidative stress-induced damage $[47,48]$. Ascorbate and glutathione are among the most important hydrophilic antioxidants in all living tissues. In this study, the effect of AMF/PGPR biofertilizer on the concentration of ascorbate, glutathione, and the glutathione precursor L-cysteine varied depending on the cultivar, tissue type, and growing season. A strong inoculation effect in relation to the concentration of glutathione, L-cysteine, and ascorbate was found in roots in 2012, but in 2013 this relationship depended on the cultivar and concerned only thiol compounds. Similarly, in Citrus tangerine roots, the GSH content was positively correlated with AMF colonization, while a significantly higher content of ascorbate in mycorrhizal plants only occurred in conditions of water deficiency [16]. An enhanced antioxidant system and glutathione-ascorbate cycle activation were observed 
in colonized barley root [49]. The leaves of inoculated apple plants consistently expressed an increasing content of glutathione in both years, while this was only true for L-cysteine in the second year of the study. Leaf ascorbate was highly year and cultivar dependent, but in both the examined years 'Chopin' exhibited a large increase in leaf ascorbate in inoculated plants. There was no AMF/PGPR effect on the ascorbate and thiol compound contents in apple peel as an indicator of nutritional value in apple. No effect has been found on ascorbic acid and glutathione in mycorrhizal tomato [50], with ascorbate concentration decreasing [13]. More examples, mainly concerning vegetables, can be found in reviews by Avio et al. [2] and Agnolluci et al. [30]. However, the results for ascorbate and less frequently tested glutathione contents depended on the species, cultivar or inoculum composition and varied considerably; an increase, no effect or decrease were noted. No research could be found on the glutathione-ascorbate cycle in AMF or co-inoculated by AMF+PGPR apple trees.

Another group of secondary metabolites with diverse biological activities in plants and benefits for human health as protective dietary agents are phenolic compounds. Potential functions, mechanisms of action, and applications of polyphenolic in relation to both plant protection and human health have recently been updated [51]. The content and distribution of phenolic compounds in apple in relation to several factors including stresses have been the subject of many studies [52-54]. According to several reports, AMF/PGPR colonization can induce both a local and systemic increase in phenolic accumulation in roots [19,55], rhizomes [25], leaves [56-58], and flower heads [56]. Salicylic acid and $\mathrm{H}_{2} \mathrm{O}_{2}$ are proposed as signaling molecules involved in these phenomena $[19,46]$. In contrast, Parada et al. [59] state that the concentration of anthocyanins in Fragaria ananassa fruit was generally higher in treatments that were not inoculated with AMF. It should be added that all the above studies were conducted in controlled greenhouse conditions. In a three-year field experiment conducted with inoculated 'Topaz' trees, the authors found that the inoculation effect on phenolic content depended on the inoculum type. Of three inocula used, only one increased the phenolic concentrations in apples [14]. Mycorrhizal inoculum resulted in an increase in total flavanols, total anthocyanins, and total polyphenols in berry skin (Vitis vinifera L.) in two experimental years [28].

In this field study, global phenolic contents were not greatly influenced by the applied inoculum in the apple cultivars tested. A significant increase in phenolics of treated plants was found in leaves, but only in 2013, and no effect was found in roots or apple peel. According to Petkovsek et al. [33], based on a two-year study leaf total phenolic content was quite stable during the growing season in both organic and integrated systems of production.

Finally, three tests (FRAP, ABTS, and DPPH) were employed to assess the impact of inoculation on TEAC antioxidant capacity in roots, leaves and apple peel. No inoculation effect on total antioxidant capacity was identified. This result was not surprising since the antioxidant capacity of plant tissue is mostly influenced by the concentration of phenolic compounds. In the presented research, the inoculum used slightly influenced the content of phenolic compounds, which was in line with the variability in the antioxidant capacity of the examined apple tissue. Phenolic compounds showed high antioxidant activity and a high positive correlation with total antioxidant activity, which has been revealed in many studies [7,60]. In the leaves of inoculated Lactuca sativa var. crispa [58], in the leaves and flower heads of artichoke plants [56], in the rhizome of Curcuma longa L. [25] and in tomato fruit [13], a significant increase in both phenolic content and antioxidant activity was reported. In another study, effective microorganisms (EM) were applied for apple orchard protection [7], with the results showing that they increase the content of polyphenols as well as boost antioxidant activity in apples.

\section{Conclusions}

The results of the present study confirmed the induction of antioxidant machinery due to AMF/PGPR inoculation of apple trees. However, the antioxidant metabolism was highly 
dependent on tissue type. In the apple cultivars tested, the greatest effect on antioxidant status was found in roots and then leaves, but this was almost negligible in fruit. Roots were influenced most by inoculum applied in the first growing season, while this was the case for leaves in the following season. Significant differences between the inoculated and control plants were found for root glutathione reductase (GR) and catalase (CAT) activity, as well as glutathione and ascorbate contents; root phenolics were not influenced by inoculation. In the case of leaves, the effect of inoculation on GR activity was revealed in the first growing season, while for global phenolics in the second season, and only the concentration of glutathione was significantly higher in the leaves of inoculated trees in both growing seasons. Leaf ascorbate content and CAT activity were not influenced by the microbial inoculation. The control and inoculated trees expressed a similar total antioxidant capacity, irrespective of the tissue type tested. Furthermore, the response of the cultivars to inoculation varied and also changed in consecutive growing seasons. Cultivar differences in the mean antioxidant content/activity were variable depending on the year, tissue type and component tested which may indicate the various dynamics of these compounds with respect to environmental conditions in the tested varieties. Based on this study, it is likely that the effect of microbial inoculum as a tool for enhancing health-promoting properties in the fruit of perennial plants is weaker than that described for vegetables where different plant organs are edible.

Supplementary Materials: The following are available online at https:/ /www.mdpi.com/article/10 .3390/app11209466/s1, Table S1: Root, leaf, and fruit total antioxidant capacity measured in ABTS, DPPH, FRAP tests: year, cultivar and bacterial-fungal inoculation effects.

Author Contributions: Conceptualization, B.E. and D.W.; methodology, B.E. and D.W.; investigation, R.Ł. and S.P.; formal analysis, R.Ł. and S.P.; supervision, B.Ł. and D.W.; visualization, S.P.; Writingoriginal draft, B.Ł.; Writing—review \& editing, B.Ł. All authors have read and agreed to the published version of the manuscript.

Funding: This research was funded by the Polish Ministry of Science and Higher Education with funds from the Institute of Horticultural Sciences.

Institutional Review Board Statement: Not applicable.

Informed Consent Statement: Not applicable.

Data Availability Statement: Data is contained within the manuscript.

Conflicts of Interest: The authors declare no conflict of interest.

\section{References}

1. Derkowska, E.; Sas Paszt, L.; Głuszek, S.; Trzciński, P.; Przybył, M.; Frąc, M. Effects of treatment of apple trees with various bioproducts on tree growth and occurrence of mycorrhizal fungi in the roots. Acta Sci. Pol. Hortorum Cultus 2017, 16, 75-83. [CrossRef]

2. Avio, L.; Turrini, A.; Giovannetti, M.; Sbrana, C. Designing the ideotype mycorrhizal symbionts for the production of healthy food. Front. Plant Sci. 2018, 9, 1089. [CrossRef]

3. Chen, M.; Arato, M.; Borghi, L.; Nouri, E.; Reinhardt, D. Beneficial services of arbuscular mycorrhizal fungi-From ecology to application. Front. Plant Sci. 2018, 9, 1270. [CrossRef] [PubMed]

4. Njeru, E.M. Exploiting diversity to promote arbuscular mycorrhizal symbiosis and crop productivity in organic farming systems. AIMS Agric. Food 2018, 3, 280-294. [CrossRef]

5. Karlidag, H.; Esitken, A.; Turan, M.; Sahin, F. Effects of root inoculation of plant growth promoting rhizobacteria (PGPR) on yield, growth and nutrient element contents of leaves of apple. Sci. Hortic. 2007, 114, 16-20. [CrossRef]

6. Cavagnaro, T.R.; Bender, S.F.; Asghari, H.R.; Van der Heijden, M.G.A. The role of arbuscular mycorrhizas in reducing soil nutrient loss. Trends Plant Sci. 2015, 20, 283-290. [CrossRef] [PubMed]

7. Kusznierewicz, B.; Lewandowska, A.; Martysiak-Zurowska, D.; Bartoszek, A. The influence of plant protection by effective microorganisms on the content of bioactive phytochemicals in apples. J. Sci. Food Agric. 2017, 97, 3937-3947. [CrossRef] [PubMed]

8. Berdeni, D.; Cotton, T.E.A.; Daniell, T.J.; Bidartondo, M.I.; Cameron, D.D.; Evans, K.L. The effects of arbuscular mycorrhizal fungal colonisation on nutrient status, growth, productivity, and canker resistance of apple (Malus pumila). Front. Microbiol. 2018, 9, 1461. [CrossRef]

9. Ortas, I. Role of mycorrhizae on mineral nutrition of fruit trees. Acta Hortic. 2018, 1217, 271-283. [CrossRef] 
10. Emmanuel, O.C.; Babalola, O.O. Productivity and quality of horticultural crops through co-inoculation of arbuscular mycorrhizal fungi and plant growth promoting bacteria. Microbiol. Res. 2020, 239, 126569. [CrossRef]

11. Santoyo, G.; Gamalero, E.; Glick, B.R. Mycorrhizal-bacterial amelioration of plant abiotic and biotic stress. Front. Sustain. Food Syst. 2021, 5, 672881. [CrossRef]

12. Latef, A.A.H.A.; Chaoxing, H. Effect of arbuscular mycorrhizal fungi on growth, mineral nutrition, antioxidant enzymes activity and fruit yield of tomato grown under salinity stress. Sci. Hortic. 2011, 127, 228-233. [CrossRef]

13. Hart, M.; Ehret, D.L.; Krumbein, A.; Leung, C.; Murch, S.; Turi, C.; Franken, P. Inoculation with arbuscular mycorrhizal fungi improves the nutritional value of tomatoes. Mycorrhiza 2015, 25, 359-376. [CrossRef]

14. Gąstoł, M.; Domagała-Światkiewicz, I. Mycorrhizal inoculation of apple in replant soils_Enhanced tree growth and mineral nutrient status. Acta Sci. Pol. Hortorum Cultus 2015, 14, 17-37.

15. Przybyłko, S.; Kowalczyk, W.; Wrona, D. The effect of mycorrhizal fungi and PGPR on tree nutritional status and growth in organic apple production. Agronomy 2021, 11, 1402. [CrossRef]

16. Wu, Q.S.; Zou, Y.N.; Xia, R.X. Effects of water stress and arbuscular mycorrhizal fungi on reactive oxygen metabolism and antioxidant production by citrus (Citrus tangerine) roots. Eur. J. Soil Biol. 2006, 42, 166-172. [CrossRef]

17. Fouad, O.M.; Essahibi, A.; Benhiba, L.; Qaddoury, A. Effectiveness of arbuscular mycorrhizal fungi in the protection of olive plants against oxidative stress induced by drought. Span. J. Agric. Res. 2014, 12, 763-771. [CrossRef]

18. Martínez-García, L.B.; Ochoa-Hueso, R.; Manrique, E.; Pugnaire, F.I. Different mycorrhizal fungal strains determine plant community response to nitrogen and water availability. J. Plant Nutr. Soil Sci. 2015, 178, 146-154. [CrossRef]

19. Zhang, R.Q.; Zhu, H.H.; Zhao, H.Q.; Yao, Q. Arbuscular mycorrhizal fungal inoculation increases phenolic synthesis in clover roots via hydrogen peroxide, salicylic acid and nitric oxide signaling pathways. J. Plant Physiol. 2013, 170, 74-79. [CrossRef]

20. Gao, W.Q.; Lü, L.H.; Srivastava, A.K.; Wu, Q.S.; Kuca, K. Effects of mycorrhizae on physiological responses and relevant gene expression of peach affected by replant disease. Agronomy 2020, 10, 186. [CrossRef]

21. Wu, Q.S.; Cao, M.Q.; Zou, Y.N.; He, X. Direct and indirect effects of glomalin, mycorrhizal hyphae, and roots on aggregate stability in rhizosphere of trifoliate orange. Sci. Rep. 2014, 4, 5823. [CrossRef]

22. Porcel, R.; Barea, J.M.; Ruiz-Lozano, J.M. Antioxidant activities in mycorrhizal soybean plants under drought stress and their possible relationship to the process of nodule senescence. New Phytologist. 2003, 157, 135-143. [CrossRef]

23. Bressano, M.; Curetti, M.; Giachero, L.; Vargas Gil, S.; Cabello, M.; March, G.; Ducasse, D.A.; Luna, C.M. Mycorrhizal fungi symbiosis as a strategy against oxidative stress in soybean plants. J. Plant Physiol. 2010, 167, 1622-1626. [CrossRef]

24. Lenoir, I.; Fontaine, J.; Sahraoui, A.L.H. Arbuscular mycorrhizal fungal responses to abiotic stresses: A review. Phytochemistry 2016, 123, 4-15. [CrossRef]

25. Dutta, S.C.; Neog, B. Accumulation of secondary metabolites in response to antioxidant activity of turmeric rhizomes coinoculated with native arbuscular mycorrhizal fungi and plant growth promoting rhizobacteria. Sci. Hortic. 2016, 204, 179-184. [CrossRef]

26. Chang, W.; Sui, X.; Fan, X.; Jia, T.; Song, F. Arbuscular mycorrhizal symbiosis modulates antioxidant response and ion distribution in salt-stressed Elaeagnus angustifolia seedlings. Front. Microbiol. 2018, 9, 652. [CrossRef] [PubMed]

27. Huang, D.; Ma, M.; Wang, Q.; Zhang, M.; Jing, G.; Li, C.; Ma, F. Arbuscular mycorrhizal fungi enhanced drought resistance in apple by regulating genes in the MAPK pathway. Plant Physiol. Biochem. 2020, 149, 245-255. [CrossRef] [PubMed]

28. Karoglan, M.; Radić, T.; Anić, M.; Andabaka, Ž.; Stupić, D.; Tomaz, I.; Mesić, J.; Karažija, T.; Petek, M.; Lazarević, B.; et al. Mycorrhizal fungi enhance yield and berry chemical composition of in field grown "Cabernet Sauvignon" grapevines ( $V$. vinifera L.). Agriculture 2021, 11, 615. [CrossRef]

29. Torres, N.; Antolín, M.C.; Goicoechea, N. Arbuscular mycorrhizal symbiosis as a promising resource for improving berry quality in grapevines under changing environments. Front. Plant Sci. 2018, 9, 897. [CrossRef] [PubMed]

30. Agnolucci, M.; Avio, L.; Palla, M.; Sbrana, C.; Turrini, A.; Giovannetti, M. Health-promoting properties of plant products: The role of mycorrhizal fungi and associated bacteria. Agronomy 2020, 10, 1864. [CrossRef]

31. Łata, B.; Przeradzka, M.; Bińkowska, M. Great differences in antioxidant properties exist between 56 apple cultivars and vegetation seasons. J. Agric. Food Chem. 2005, 53, 8970-8978. [CrossRef] [PubMed]

32. Musacchi, S.; Serra, S. Apple fruit quality: Overview on pre-harvest factors. Sci. Hortic. 2018, 234, 409-430. [CrossRef]

33. Petkovsek, M.M.; Slatnar, A.; Stampar, F.; Veberic, R. The influence of organic/integrated production on the content of phenolic compounds in apple leaves and fruits in four different varieties over a 2-year period. J. Sci. Food Agric. 2010, 90, $2366-2378$. [CrossRef] [PubMed]

34. Gosling, P.; Ozaki, A.; Jones, J.; Turner, M.; Rayns, F.; Bending, G.D. Organic management of tilled agricultural soils results in a rapid increase in colonization potential and spore populations of arbuscular mycorrhizal fungi. Agric. Ecosyst. Environ. 2010, 139, 273-279. [CrossRef]

35. Gottshall, C.B.; Cooper, M.; Emery, S.M. Activity, diversity and function of arbuscular mycorrhizae vary with changes in agricultural management intensity. Agric. Ecosyst. Environ. 2017, 241, 142-149. [CrossRef]

36. Turrini, A.; Agnolucci, M.; Palla, M.; Tomé, E.; Tagliavini, M.; Scandellari, F.; Giovannetti, M. Species diversity and community composition of native arbuscular mycorrhizal fungi in apple roots are affected by site and orchard management. Appl. Soil Ecol. 2017, 116, 42-54. [CrossRef] 
37. Cavallazzi, J.R.P.; Filho, O.K.; Sturmer, S.L.; Rygiewicz, P.T.; Matos de Mendonca, M. Screening and selecting arbuscular mycorrhizal fungi for inoculating micropropagated apple rootstocks in acid soils. Plant Cell Tissue Organ Cult. 2007, 90, 117-129. [CrossRef]

38. Ridgway, H.J.; Kandula, J.; Stewart, A. Arbuscular mycorrhiza improve apple rootstock growth in soil conducive to specific apple replant disease. New Zealand Plant Prot. 2008, 61, 48-53. [CrossRef]

39. Raj, H.; Sharma, S.D. Integration of soil solarization and chemical sterilization with beneficial microorganisms for the control of white root rot and growth of nursery apple. Sci. Hortic. 2009, 119, 126-131. [CrossRef]

40. Łata, B. Relationship between apple peel and the whole fruit antioxidant content: Year and cultivar variation. J. Agric. Food Chem. 2007, 55, 663-671. [CrossRef]

41. Stefaniak, J.; Łata, B. Actinidia arguta leaf as a donor of potentially healthful bioactive compounds: Implications of cultivar, time of sampling and soil $\mathrm{N}$ level. Molecules 2021, 26, 3871. [CrossRef] [PubMed]

42. Medina, M.B. Determination of the total phenolics in juices and superfruits by a novel chemical method. J. Funct. Foods 2011, 3 , 79-87. [CrossRef]

43. Escarpa, A.; González, M. High-performance liquid chromatography with diode-array detection for the determination of phenolic compounds in peel and pulp from different apple varieties. J. Chromatogr. A 1998, 823, 331-337. [CrossRef]

44. Baum, C.; El-Tohamy, W.; Gruda, N. Increasing the productivity and product quality of vegetable crops using arbuscular mycorrhizal fungi: A review. Sci. Hortic. 2015, 187, 131-141. [CrossRef]

45. Nadeem, S.M.; Ahmad, M.; Zahir, Z.A.; Javaid, A.; Ashraf, M. The role of mycorrhizae and plant growth promoting rhizobacteria (PGPR) in improving crop productivity under stressful environments. Biotechnol. Adv. 2014, 32, 429-448. [CrossRef]

46. Zhu, H.; Zhang, R.; Chen, W.; Gu, Z.; Xie, X.; Zhao, H.; Yao, Q. The possible involvement of salicylic acid and hydrogen peroxide in the systemic promotion of phenolic biosynthesis in clover roots colonized by arbuscular mycorrhizal fungus. J. Plant Physiol. 2015, 178, 27-34. [CrossRef]

47. Fenech, M.; Amaya, O.; Valpuesta, V.; Botella, M.A. Vitamin C content in fruits: Biosynthesis and regulation. Front. Plant Sci. 2019, 9, 2006. [CrossRef]

48. Hasanuzzaman, M.; Bhuyan, M.H.M.B.; Anee, T.I.; Parvin, K.; Nahar, K.; Al Mahmud, J.; Fujita, M. Regulation of AscorbateGlutathione pathway in mitigating oxidative damage in plants under abiotic stress. Antioxidants 2019, 8, 384. [CrossRef]

49. Waller, F.; Achatz, B.; Baltruschat, H.; Fodor, J.; Becker, K.; Fischer, M.; Heier, T.; Ralph Hückelhoven, R.; Neumann, C.; Wettstein, D.; et al. The endophytic fungus Piriformospora indica reprograms barley to salt-stress tolerance, disease resistance, and higher yield. Proc. Natl. Acad. Sci. USA 2005, 102, 13386-13391. [CrossRef]

50. Giovannetti, M.; Avio, L.; Barale, R.; Ceccarelli, N.; Cristofani, R.; Iezzi, A.; Mignolli, F.; Picciarelli, P.; Pinto, B.; Reali, D.; et al. Nutraceutical value and safety of tomato fruits produced by mycorrhizal plants. Br. J. Nutr. 2012, 107, 242-251. [CrossRef]

51. Khalid, M.; Rahman, S.; Bilal, M.; Dan-feng, H. Role of flavonoids in plant interactions with the environment and against human pathogens-A review. J. Integr. Agric. 2019, 18, 211-230. [CrossRef]

52. Łata, B.; Trampczyńska, A.; Pacześna, J. Cultivar variation in apple peel and whole fruit phenolic composition. Sci. Hortic. 2009, 121, 176-181. [CrossRef]

53. Volz, R.K.; Mc Ghie, T.K. Genetic variability in apple fruit polyphenol composition in Malus $x$ domestica and Malus sieversii germplasm grown in New Zealand. J. Agric. Food Chem. 2011, 59, 11509-11521. [CrossRef] [PubMed]

54. Skłodowska, M.; Mikiciński, A.; Wielanek, M.; Kuźniak, E.; Sobiczewski, P. Phenolic profiles in apple leaves and the efficacy of selected phenols against fire blight (Erwinia amylovora). Eur. J. Plant Pathol. 2018, 15, 213-228. [CrossRef]

55. Li, J.F.; He, X.H.; Li, H.; Zheng, W.J.; Liu, J.F.; Wang, M.Y. Arbuscular mycorrhizal fungi increase growth and phenolics synthesis in Poncirus trifoliata under iron deficiency. Sci. Hortic. 2015, 183, 87-92. [CrossRef]

56. Ceccarelli, N.; Curadi, M.; Martelloni, L.; Sbrana, C.; Picciarelli, P.; Giovannetti, M. Mycorrhizal colonization impacts on phenolic content and antioxidant properties of artichoke leaves and flower heads two years after field transplant. Plant Soil 2010, 335, 311-323. [CrossRef]

57. Chen, S.; Jin, W.; Liu, A.; Zhang, S.; Liu, D.; Wang, F.; Lin, X.; He, C. Arbuscular mycorrhizal fungi (AMF) increase growth and secondary metabolism in cucumber subjected to low temperature stress. Sci. Hortic. 2013, 160, 222-229. [CrossRef]

58. Avio, L.; Sbrana, C.; Giovannetti, M.; Frassinetti, S. Arbuscular mycorrhizal fungi affect total phenolics content and antioxidant activity in leaves of oak leaf lettuce varieties. Sci. Hortic. 2017, 224, 265-271. [CrossRef]

59. Parada, J.; Valenzuela, T.; Gómez, F.; Tereucán, G.; García, S.; Cornejo, P.; Winterhalter, P.; Ruiza, A. Effect of fertilization and arbuscular mycorrhizal fungal inoculation on antioxidant profiles and activities in Fragaria ananassa fruit. J. Sci. Food Agric. 2019, 99, 1397-1404. [CrossRef]

60. Teleszko, M.; Wojdyło, A. Comparison of phenolic compounds and antioxidant potential between selected edible fruits and their leaves. J. Funct. Foods 2015, 14, 736-746. [CrossRef] 